Determination of the geoid of central highlands in Sri

Lanka

by

Herath Mudiyanselage Indika Prasanna

Thesis submitted to the University of Sri Jayewardenepura for the award of the Degree of Master of Philosophy in Geodesy on $19-09-2008$. 
"The work described in this thesis was carried out by me under the supervision of Professor D.A.Tantrigoda and a report on this has not been submitted in whole or in part to any University or any other Institution for another Degree/Diploma".

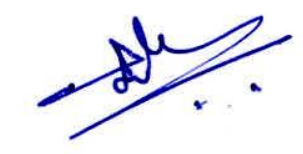

19-09-2008

Signature of the candidate

Date 
"I certify that the above statement made by the candidate is true and that this thesis is suitable for submission to the University for the purpose of evaluation".

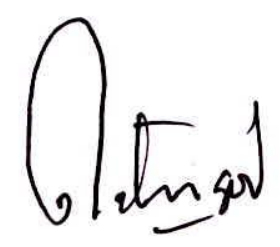

19-09-2008

Signature of supervisor

Date

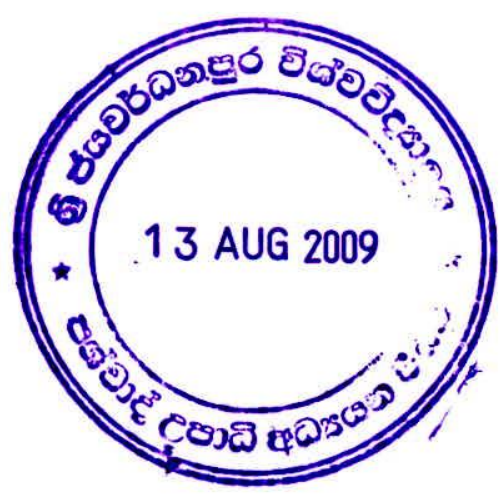




\section{Determination of the geoid of central highlands in Sri Lanka}

by

\section{Herath Mudiyanselage Indika Prasanna}

\section{ABSTRACT}

Geoidal undulations over two hundred GPS stations have been interpolated using least-squares surface fitting technique to determine the geoid of central highlands in Sri Lanka. However, it is not possible to interpolate these points directly to prepare a detailed map of geoidal undulations as its component due to the topography is rapidly varying making the interpolation inaccurate. Gravity potential and the undulation of the local geoid due to the topography have been calculated separately using a topographic model and removed from the above available geoidal undulations. Resulting data were interpolated using a leastsquares technique and the Bouguer co-geoid was determined as two different cases. In the first case, a single mathematical surface was fitted using all reduced geoidal undulations situated over the entire Sri Lanka. In the second case, three surface polynomials were employed to determine the Bouguer co-geoid for the same area. Finally, the geoidal undulations due to the topography were added back to each of the above mathematical surface to create a detailed map of the geoid of central highlands in Sri Lanka. In both cases, local positive geoidal surfaces superimposed on a large negative regional surface have been obtained and the minimum value of the geoidal undulation is about $-92.05 \mathrm{~m}$ in the vicinity of Piduruthalagala peak. 


\section{Acknowledgements}

I wish to express my special gratitude to the Research Grants Committee of Sabaragamuwa University of Sri Lanka for providing necessary funds to complete this research.

First of all I would like to express my sincere gratitude to my supervisors Professor D.A.Tantrigoda and Mr. S.T.Herat for their guidance and advices they offered me in shaping-up my research work.

My heartfelt gratitude extends to the members of academic and non-academic staff of the University of Sri Jayewardenepura for giving encouragements and providing facilities throughout the research period.

My gratitude extends to the members of the academic staff of the Sabaragamuwa University for encouraging me towards higher studies.

Above all, I wish to thank my wife, my mother and father for their morale support, without which I will never be able to complete this study. 


\section{Table of contents}

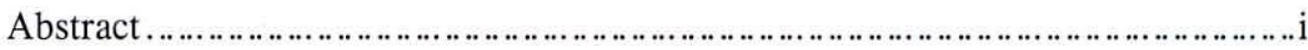

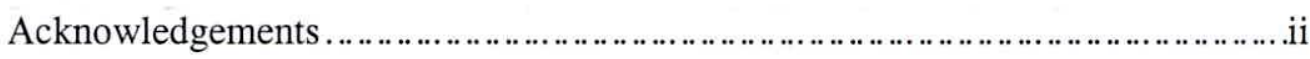

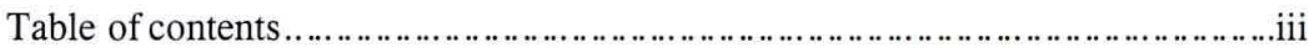

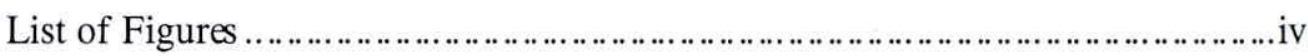

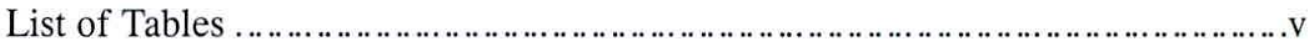

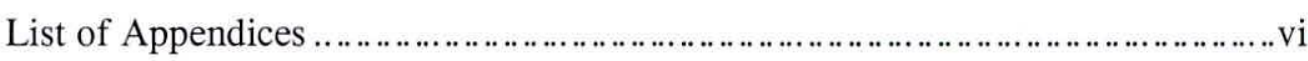

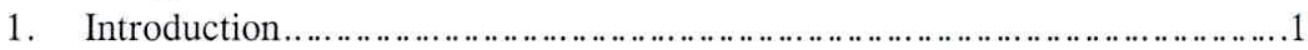

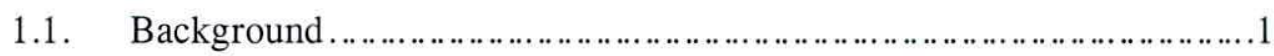

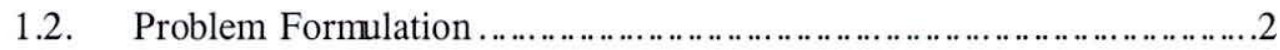

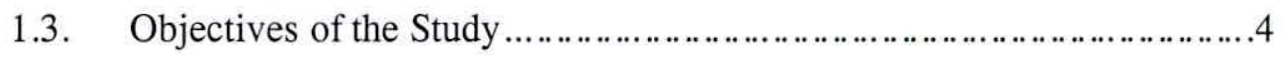

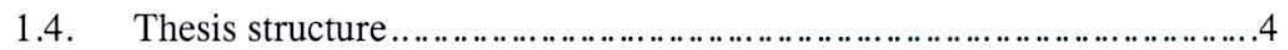

2. Review of the methods of determination of the geoid .............................6

2.1. Gravimetric geoid determination .............................................6

2.2. The Astrogeodetic determination of the geoid................................. 10

2.3. The least-squares surface fitting method .......................................11

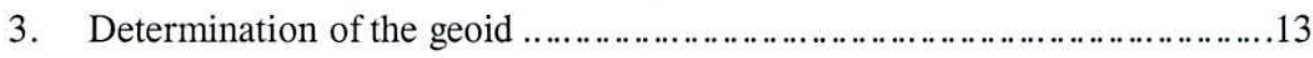

3.1. Theoretical background ..........................................................13

3.2. Topographic model using cylindrical sections................................15

3.3. Topographic model using polygonal sections ................................17

3.4. Topographic model using digitized contours...............................20

3.4.1. Some justifications to the topographic model..........................21

3.5. Surface fitting and geoid maps .................................................25

3.5.1. Determination of geoid using a single mathematical surface .......26

3.5.2. Determination of geoid using three surface polynomials ............29

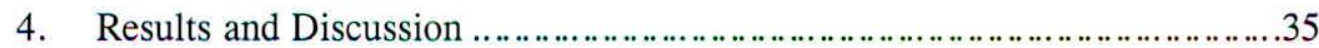

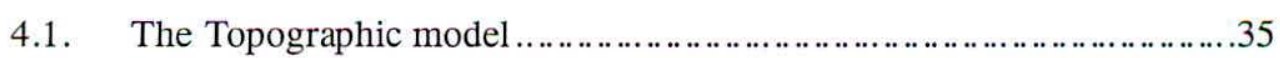

4.2. Least-squares surface fitting and Bouguer co-geoid ...........................35

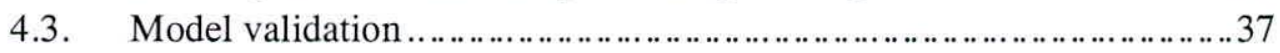

5. Conclusions and Recommendations .................................................40

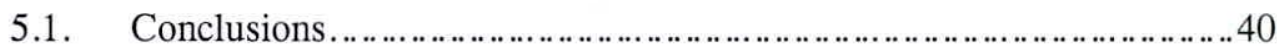

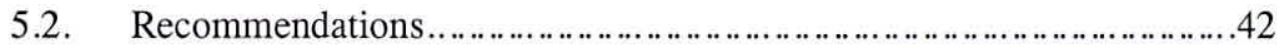

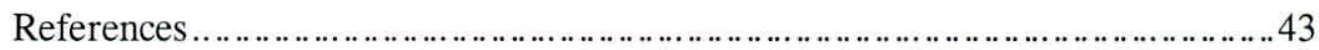

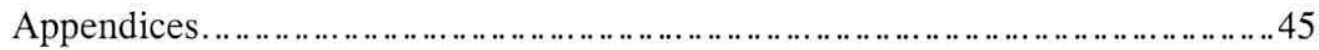




\section{List of Figures}

Figure 1-1: Geoid and reference ellipsoid .................................................3

Figure 3-1: Cylindrical laminae (real coordinates $(200000 \mathrm{~N}, 200000 \mathrm{E})$ taken as $(0$,

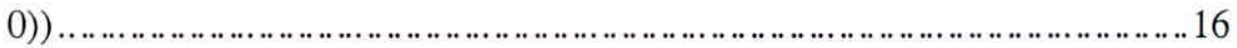

Figure 3-2: Topographic undulation map of Sri Lanka (created using the model of

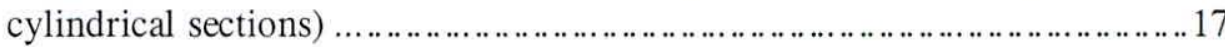

Figure 3-3: Polygonal laminae (real coordinates $(200000 \mathrm{~N}, 200000 \mathrm{E})$ taken as $(0$,

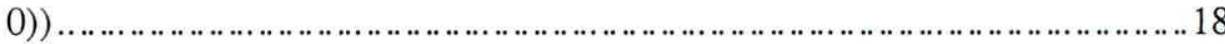

Figure 3-4: Topographic undulation map of Sri Lanka (created using the model of

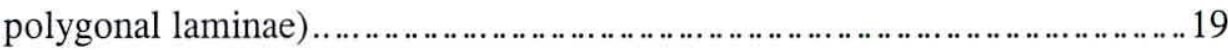

Figure 3-5: The effect on geoidal undulation due to topography in Sri Lanka ......21

Figure 3-6: A TIN model showing the actual topography in Sri Lanka (Elevation

ranges in meters) ....................................................................22

Figure 3-7: The applied topographic model of Sri Lanka (Elevation ranges in meters)

Figure 3-8: The color pattern shows the undulation range of Bouguer co-geoid and

the two areas which have rapid variations (Undulation range in imeters) .....25

Figure 3-9: The degree of the polynomials and their RMS values of residuals....26

Figure 3-10: The region of Sri Lanka where geoidal surface to be determined.....27

Figure 3-11: The Bouguer co-geoid map of central highlands ........................28

Figure 3-12: Geoid map of central highlands using single polynomial method ....29

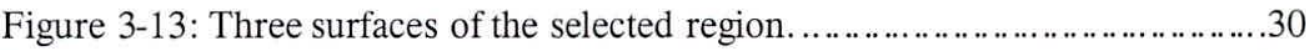

Figure 3-14: The degree of the polynomials and their RMS values of residuals of

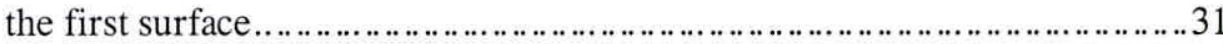

Figure 3-15: The surface views of the three regions (Elevation ranges in millimeters)

Figure 3-16: The Bouguer co-geoid map of central highlands using three surface

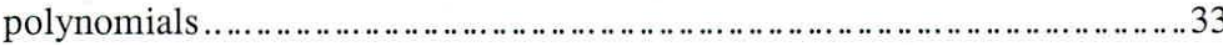

Figure 3-17: Geoid map of central highlands using three surface polynomials ....34

Figure 41: The residuals of single surface fitting method for the entire data set .36

Figure 42: The residuals of single surface fitting method for the selected region 36

Figure 4-3: The residuals of the second case which uses three surface polynomials

Figure 44: The positions of data points used for model validation ...................38

Figure $0-1$ : Geometrical elements involved in the computation of gravity potential caused by a three dimensional body 


\section{List of Tables}

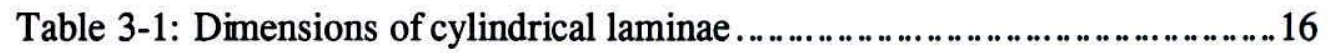

Table 3-2: Dimensions of polygonal laminae ...............................................18

Table 3-3: The geometrical configuration of the topographic model ..................24

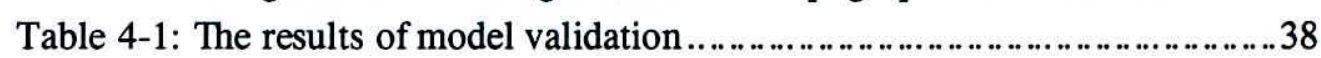




\section{List of Appendices}

Appendix 1- Derivation of the analytical expressions .45

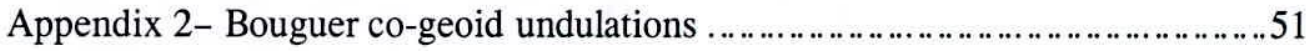

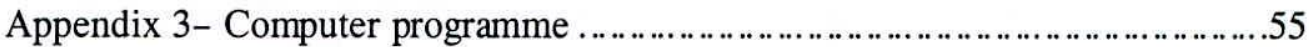




\section{Introduction}

\subsection{Background}

The initial survey activity for a country is the establishment of its geodetic control network. Before the advent of new technology, triangulation was the only process that was available to establish control points in Sri Lanka. When Electromagnetic Distance Measurement (EDM) technology and Global Positioning System (GPS) were introduced, this became a relatively convenient task. In 1992, the revision work of the geodetic control network was commenced using GPS and in 1994 the country's first high accurate GPS network was established.

Although, the planimetric accuracy of control points provided by triangulation was improved by use of the GPS technology, the heights of triangulation stations from the Mean Sea Level (MSL) had been calculated employing the reciprocal vertical angle method. The MSL extended for the whole of the earth is generally referred to as the geoid. In view of the inaccuracy of this method, the method of precise levelling, as the procedure to determine heights between points on the Earth surface, had been undertaken since 1909 by the survey department of Sri Lanka. The primary levelling network covered the entire country and was then densified by establishing secondary, tertiary and minor levelling networks to provide height control for all the development projects in Sri Lanka. 
The height measured from the MSL, usually defined as the orthometric height, is an important parameter in any survey. Many countries tend to use the GPS technology for obtaining orthometric heights as the levelling procedure is time consuming. However GPS technology can not be used directly for obtaining orthometric heights. In the GPS system, heights are measured from an ellipsoidal surface called World Geodetic System 1984 (WGS 84) which has been defined using relevant parameters. Therefore, measured ellipsoidal heights using the GPS technique require the position of the geoid relative to the ellipsoid in order to determine the corresponding orthometric heights.

As explained above, computation of orthometric heights is still being a problem unless the precise geoidal surface is known.

\subsection{Problem Formulation}

As indicated above, the geoid is usually determined relative to the WGS 84 reference ellipsoid. The relationship between the geoid and the reference ellipsoid is given by their linear separation called geoidal undulation $(N)$ and the deflection of the vertical $(\theta)$, the angular deviation between normal to the ellipsoid and that of to the geoid. Since deflection of the vertical is very small and can be neglected for most practical purposes, geoidal undulation is the major factor representing the relationship between the geoid and the ellipsoid. Therefore, the relationship between the ellipsoidal height $(h)$, the corresponding orthometric height $(H)$ and the geoidal undulation $(N)$ can be expressed using the simple equation,

$$
h=H+N
$$




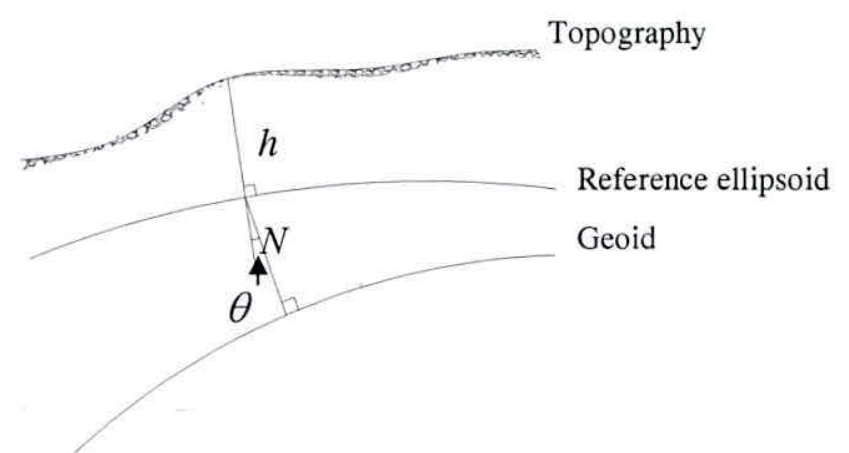

Figure 1-1: Geoid and reference ellipsoid

At present in Sri Lanka, geoidal undulations are available for little over 200 GPS stations and 50 trignometrical stations situated in various parts of Sri Lanka. Based on the available data, geoidal surface can be interpolated, for an area where there are no large topographical features, using the least-squares surface fitting technique. Surface polynomials of different degrees are fitted to the above geoidal undulations and the one, which gives the least residuals, is selected as the best surface polynomial that approximates to the geoid. After determining the best geoidal surface over the entire country, geoidal undulation can be obtained as a function of position on the surface. Hence the orthometric heights can directly be computed by direct substitution in the above equation.

Geoidal undulations depend on the topography as well as sub-surface mass anomalies. The component that depends on mass anomalies (Bouguer co-geoid) has smooth variations as it is being caused by deep seated sources. However, the component that depends on the topography has large variations especially in areas where there are rugged topography such as the central highlands in Sri Lanka. 
Therefore, geoidal undulations can not be determined accurately by mere interpolation of available undulations mentioned above due to problem that will be caused by the component which has rapid variations. Hence, first the component that depends on the topography has been removed from the available undulations before fitting a mathematical surface. After determining the Bouguer co-geoid, the removed topographic effect of undulation has been added back to obtain the total geoidal undulation.

\subsection{Objectives of the Study}

1 Determination of the mathematical surface which approximates to the geoid of Sri Lanka.

2 Computation of orthometric heights using GPS derived ellipsoidal heights with geoidal undulations.

\subsection{Thesis structure}

Chapter 1 gives an introduction to outline the general context of the work, including the background of the study, the research problem solved in this research work, objectives of the study and research methodologies.

Chapter 2 deals with the presentation of the literature review on various methods of determination of the geoid. This includes gravimetric, astrogeodetic and leastsquares surface fitting methods. 
Chapter 3 describes the methodology of determination of the geoid of Sri Lanka including the theoretical background, the construction of various topographic models and different methods of surface fitting technique.

Chapter 4 focuses on the results obtained by the two different surface fitting techniques and the comparison of their accuracies using a model validation.

Chapter 5 gives the conclusions and recommendations for future work. 


\section{Review of the methods of determination of the geoid}

\subsection{Gravimetric geoid determination}

Geoid is defined as the equipotential surface of the earth's gravity field coinciding with the mean sea levels of the oceans. Averaging the ocean surface over a time (at least over one year) or modeling ocean tides provides mean sea levels (MSL) for the corresponding time interval (Torge, 2001).

According to Newton's law of gravitation, the Earth's effective gravitational potential $W$ at point on the surface of Earth is expressed by the formula:

$$
W=V+\phi=G \iiint_{v} \frac{\rho d v}{r}+\frac{(l \omega)^{2}}{2}
$$

Where $V$ is gravitational potential of the earth;

$\phi$ is centrifugal potential of the earth;

$d v$ is an element of unit volume inside the earth of total volume $v$;

$r$ is the distance from the mass element to a specified point;

$\omega$ is the angular velocity of the earth; and

$l$ is the perpendicular distance from the axis of rotation 